\title{
Discrimination of the number of sequential events by rats
}

\author{
DONNA M. FERNANDES and RUSSELL M. CHURCH \\ Brown University, Providence, Rhode Island
}

\begin{abstract}
Ten rats were trained to press a right lever following two successive sounds and to press a left lever following four successive sounds. Three sound sequences were used in initial discrimination training, such that reliable classification could not be based on the duration of each sound, the interval between sounds, or the total duration of the sound sequence. Classification of seven novel sound sequences suggested that the animals were either using number or the sum of sound durations in a sequence as the relevant cue. When this total sound duration was put in conflict with number, rats classified by number. The conclusion was that rats can discriminate number, even when temporal cues are controlled.
\end{abstract}

The problem was to determine whether the number of successive events (stimuli or responses) could serve as an effective discriminative stimulus. Although earlier research on fixed-ratio (FR) performance (e.g., Ferster \& Skinner, 1957) involved variation in the number of responses necessary for reinforcement, Mechner (1958) was the first to attempt to identify the number of responses as an effective discriminative stimulus. In this procedure the press of a left lever (left leverpress) by a rat was reinforced only after at least $\mathbf{N}$ successive right leverpresses, with values of $\mathrm{N}$ over blocks of sessions at 4,8 , 12 , or 16 responses. The probability of a left leverpress as a function of the number of prior right leverpresses was fairly symmetrical on a linear scale, with a maximum probability near $\mathbf{N}$. As $\mathbf{N}$ increased $(4,8,12,16)$, variability of the distribution increased. The effective variable in Mechner's experiment might have been how long the animal pressed the right lever, not the number of right leverpresses. If the animal pressed on the right lever at a fairly steady rate, as usually occurs in FR schedules, the number of right leverpresses would be completely confounded with the time elapsed since the first right leverpress. Functions similar to those obtained by Mechner occur when reinforcement is contingent on a response after a particular time in the peak procedure (Roberts, 1981). Therefore, although counting may account for Mechner's data, a timing mechanism is also plausible.

One way to separate time and number is to use a treatment that changes response rate. Mechner

This research was supported by NSF Grant BNS 79-04792. We thank Warren $H$. Meck for assistance and advice on this experiment. Reprints may be obtained from Russell $\mathbf{M}$. Church, Department of Psychology, Brown University, Providence, Rhode Island 02912. and Guevrekian (1962) found that an increase in water deprivation increased response rate but did not affect the relation between the probability of a left leverpress and the number of preceding right leverpresses. Thus, they concluded that the left presses were controlled by number, not time. Even if rapid learning of a new temporal criterion is ruled out, however, subjective time could be the determiner of the left leverpress. An increase in deprivation might increase the speed of an internal clock, given Roberts' (1981) finding that prefeeding increased the maximum of the function relating signal duration to probability of a response in the peak procedure, a result that can be interpreted as slowing the rate of an internal clock.

In an investigation using similar logic, Laties (1972) used drugs to change the response rate in a Mechner procedure. Haloperidol decreased response rate. Thus, it increased the optimal time to switch, but it did not affect the number of responses before switching. Again, even without rapid learning of a new temporal discrimination, the experiment would show only that time was irrelevant if subjective time accurately reflected objective time. Maricq, Roberts, and Church (1981) found that methamphetamine decreased the point of indifference in a temporal estimation procedure in a manner consistent with about a $10 \%$ increase in clock rate; Maricq and Church (in press) replicated this result and found that haloperidol increased the point of indifference in a manner consistent with a decrease in clock rate. In Laties's experiment, if haloperidol decreased the speed of the clock in the same proportion as it did the response rate, and the animal maintained a constant subjective time criterion, the number of responses before a switch to the left lever response would not change. Of course, the counting interpretation of these data is simpler than the timing in- 
terpretation and it has the important advantage of making a quantitative prediction, but these arguments show that the general approach of these experiments is inconclusive.

The most direct demonstration of the counting interpretation of performance in the Mechner procedure was conducted by Wilkie, Webster, and Leader (1979). They replicated some of the rat results with pigeons, but following each keypeck they added a random blackout period during which the animal did not respond. The function was equivalent when the blackout duration was fixed so that either timing or counting could occur, and when it varied randomly so that, they argued, only counting could control accurate responding. In fact, even with these random-duration blackouts, the probability of reinforcement was related to the time elapsed since a trial began.

There is, however, one additional problem with any response counting experiment. Suppose each response duration is about the same and the rat sums these durations. Then the animal might be timing rather than counting. It is reasonable to assume that rats can sum the durations of discrete signals, because this is the essential finding of the "gap" experiments in which rats ran their clocks during a signal but stopped them during signal-off periods (Roberts $\&$ Church, 1978). This is a concern in various response counting procedures (e.g., Dodd, 1980; Hobson, 1975; Hobson \& Newman, 1981; Plisk off \& Goldiamond, 1966; Rilling, 1967). Although it may be difficult to discredit this idea in the case of counting responses, the test is straightforward in the case of counting stimuli. Since the experimenter can independently control the number of stimuli presented, and the duration of each, the sum of stimulus durations is not determined by the number of stimuli.

Animals that were trained to discriminate between different sequences of auditory or visual stimuli in previous studies have had the opportunity to use various temporal cues instead of number. Woodrow (1929, cited in Honigmann, 1942) trained rhesus monkeys to distinguish a sequence of two sounds from a sequence of three sounds, but when the total time required for each sequence was made the same, the discrimination was lost. Similarly, when Douglas and Whitty (1941) equated for total sequence duration, the discrimination of one vs. two successive flashes of light by four baboons was severely hampered. In the present experiment, the sound sequences used in initial training and in later testing were designed to minimize reliance on extraneous temporal cues.

\section{METHOD}

\section{Subjects}

The subjects were 10 experimentally naive male albino Norway rats (Charles River CD), approximately 120 days old at the start of training. The animals were kept in individual metal cages (Wahmann Mfg. Co., Baltimore, Md.), given unlimited access to water, and maintained on daily rations of Charles River Rat Formula (about $14 \mathrm{~g}$ ) mixed with water and delivered at the end of each session.

\section{Apparatus}

Ten lever boxes $(23 \times 20 \times 22 \mathrm{~cm})$ were used. The roof and the two side walls were transparent acrylic; the front and back walls were aluminum. The floor was constructed of 16 parallel stainless steel bars. Two retractable stainless steel levers projected through the front panel on either side of the food cup. The levers in Boxes 1.6 were $1.6 \times 4.6 \mathrm{~cm}$ and were located $3.8 \mathrm{~cm}$ above the floor (Gerbrands Model 6311). The levers in Boxes 7-10 were 2.5 $\times$ $5.0 \mathrm{~cm}$ and were located $5.0 \mathrm{~cm}$ above the floor (BRS/LVE Model 123-07). A pellet dispenser (Gerbrands Model D-1 or Davis Scientific Instruments Model PD-104) delivered 45-mg Noyes Precision food pellets through an opening in the front wall to a food cup. A $140-\mathrm{ml}$ glass water bottle, at least half full, hung from the back wall of the chamber. Each lever box was housed in an insulation-board chamber designed to block outside light and sound. A houselight (7.5 W in Boxes 1-6 and 6.0 W in Boxes 7-10) was the only source of light and was kept on throughout all sessions. Each chamber had a ventilating fan and a small observation window.

\section{Procedure}

For pretraining, each rat received one session of combined magazine and lever training. Single food pellets were delivered once each minute for 10 min (magazine training), and, in addition, leverpresses were reinforced with food. First the left lever was inserted and 10 responses were reinforced; then only the right lever was inserted and 10 more responses were reinforced. This alternation continued until 60 leverpresses had occurred. The next two sessions consisted of lever training only.

The subjects were trained (Days 1-30) to press the right lever following a sequence of two bursts of white noise and to press the left lever following a sequence of four bursts of white noise. Each burst was $.2 \mathrm{sec}$ in duration. When two successive bursts were presented, they were separated by intersound intervals (ISI) of either .8 or $2.8 \mathrm{sec}$; when four successive bursts were presented, the individual sounds were separated by an ISI of $.8 \mathrm{sec}$. The total sequence duration was defined as the time from the onset of the first soundburst to the termination of the last one, including ISIs. The total sequence duration of the two-sound sequences was either $1.2 \mathrm{sec}$ (2-short) or $3.2 \mathrm{sec}$ (2-long), and the total sequence duration of the four-sound sequence was $3.2 \mathrm{sec}$ (4-long).

On each trial, one of the three sequences was presented. The 2-short sequence was presented with a probability of .25 , the 2 -long sequence was presented with a probability of .25 , and the 4-long sequence was presented with a probability of .50. Following a sound sequence, both levers were inserted into the box. If the subject responded correctly, food was delivered and both levers were withdrawn; if the subject responded incorrectly, both levers were withdrawn and the same sound sequence was presented again on the next trial (correction procedure). Intertrial intervals were geometrically distributed with a mean of $30 \mathrm{sec}$ and a minimum of .1 sec. All sessions in the experiment were $1 \mathrm{~h} 50 \mathrm{~min}$ long.

Testing (Days 31-35) were identical to training except that seven novel sequences were presented on half the trials, each with equal probability $(p=.0714)$. These sequences varied along the dimensions of number of sounds (two or four) and of intersound interval $(.8,1.3,1.8,2.3$, or $2.8 \mathrm{sec})$. The three sequences used in training were now presented with the probabilities $p(4-\mathrm{long})=.25$, $\mathrm{p}(2$-short $)=.125$, and $\mathrm{p}(2$-long $)=.125$. Responses to the seven novel sequences were not reinforced. There were no correction trials during this or the subsequent phase of the experiment.

Training on 10 conditions (Days 36-45) was identical to testing except that correct responses to all 10 sequences were reinforced.

Following a 7-week interruption, the rats were retrained on all 10 signals (Days 46-55). After 5 days of retraining, on three- 
fourths of the trials the conditions were the same, but on the remaining one-fourth of the trials one of four novel sequences was now presented with a probability of .0625 . These sequences also varied along the dimensions of number of sounds (two or four) and intersound interval (.8 or $2.8 \mathrm{sec}$ ). Each burst of white noise was $.4 \mathrm{sec}$ in duration when two bursts were presented but $.1 \mathrm{sec}$ in duration when four bursts were presented. Responses to the four novel sequences were not reinforced. Correct responses to the other 10 sequences continued to be reinforced.

\section{RESULTS}

The rats learned to discriminate the 4-sound sequence from the 2-sound sequences. Initially, the probability of a left ("many") response was about .5. The rats increased their proportion of "many" responses to the 4-sound sequence, and they decreased their proportion of "many" responses to both of the 2-sound sequences. On the last 5 days of training, the probabilities of a "many" response were $.74, .16$, and .20 for the 4-long, 2-long, and 2-short groups, respectively. Throughout most of training, the rats responded correctly most often to the 2-sound sequence with the short ISI. During the last 15 days of training, there was a significant difference in the proportion of correct responses among the three sequences $[\mathrm{F}(2,18)=12.7, \mathrm{p}<.01]$. The 2 -short sequence was classified correctly more often than either the 2-long or the 4-long sequence (Tukey HSD test, $\mathrm{p}<.05$ ).

Figure 1 shows the first 5 days of testing on 10 conditions, in which responses to the seven novel sequences were not reinforced. On the 1st day of testing (top panel), the major determinant of classification of the novel sequences was the number of sounds, rather than the ISI. The sequences consisting of four soundbursts were categorized as "many" much more often than the sequences consisting of two soundbursts $[F(1,36)=197, p<.001]$; there was no significant relationship between the ISI and the proportion of "many" responses $[F(4,36)=.88$, $\mathrm{p}>.05]$.

Figure 1 also shows the decline in performance on the 4-sound sequence during the 5 days of testing, particularly for the unreinforced 4-sound sequences with long ISIs. On the 5th day of testing, the proportion of "many" responses following the 4-sound sequence with the longest ISI $(2.8 \mathrm{sec})$ was indistinguishable from the proportion following the 2-sound sequence with the same ISI $[t(9)=1.34, p>$ $.10]$; classification of the other 4-sound sequences remained significantly above the corresponding 2 -sound sequences, with ISI $=2.3 \mathrm{sec}[\mathrm{t}(9)=2.32$, $\mathrm{p}<.05]$.

Results from training for all 10 cases are shown in Figure 2. Reinforcement of correct responses to the novel sequences markedly improved the discrimination. The median probability of a "many" re-

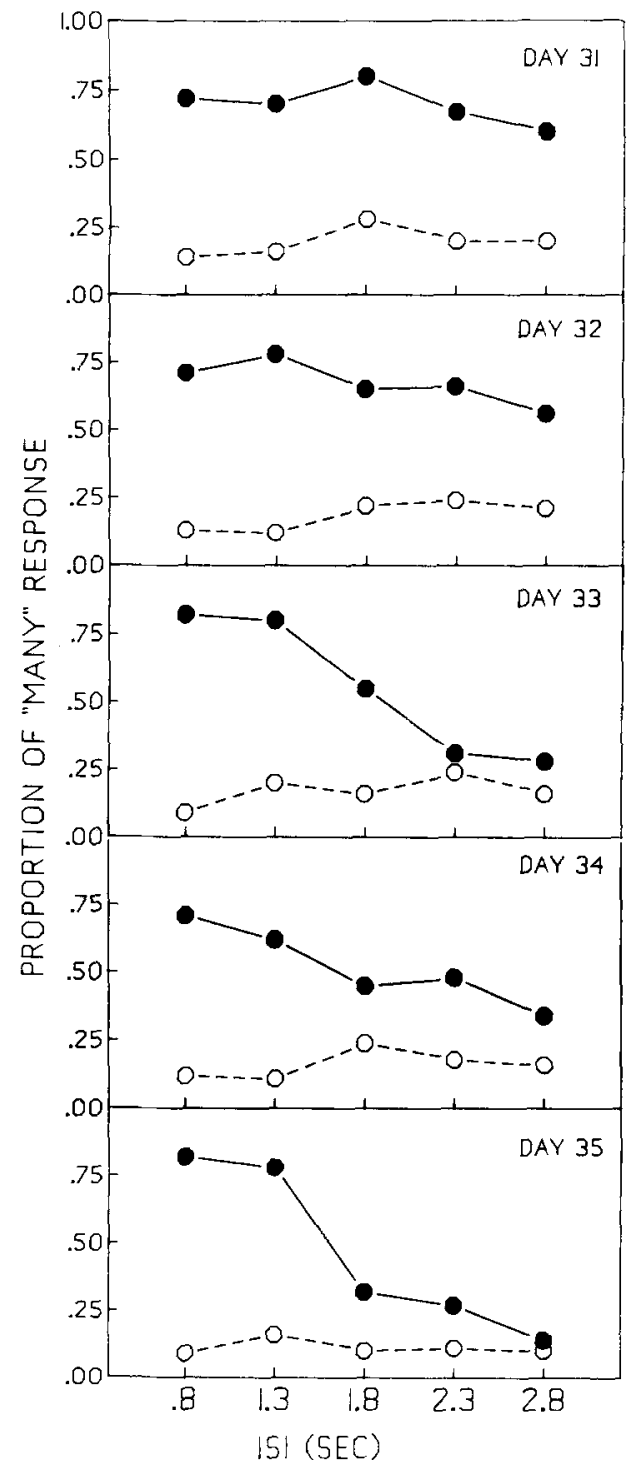

Figure 1. The first 5 days of testing on the 10 conditions. Median proportion of "many" responses as a function of intersound interval (ISI). Solid circles indicate sequences of four sounds; open circles indicate sequences of two sounds. (Figures were drawn with a program developed by Hayes, 1981.)

sponse following a 4-sound sequence was .81 and following a 2-sound sequence was .11 on the first 5 days of training and .92 (4-sound sequence) and .06 (2-sound sequence) on the next 5 days of training. The slopes of lines relating the proportion of "many" responses to ISI for the 2-sound sequences were not significantly different from zero on Days 36-40 $[\mathrm{t}(9)=2.20, p>.05]$ or on Days $41-45$ $[\mathrm{t}(9)=1.19, \mathrm{p}>.05]$; however, there were a slight, but significant, negative slope for the 4-sound sequences on Days $36-40[t(9)=2.29, p<.05]$ and a slight, but significant, positive slope for the 4-sound sequence on Days 41-45 $[\mathrm{t}(9)=5.14, \mathrm{p}<.0011$. On 


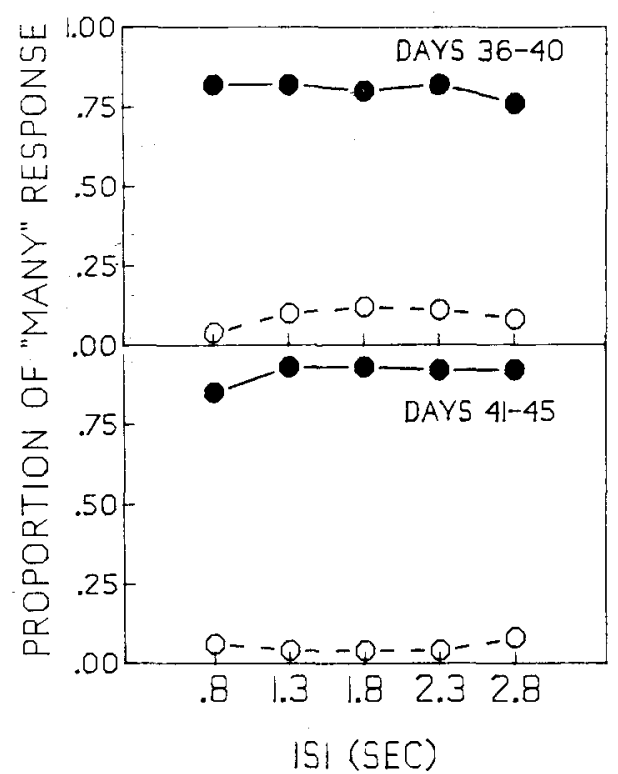

Figure 2. Training on the 10 conditions (i.e., correct responses to the novel sequences now reinforced). Median proportion of "many" responses as a function of intersound interval for the first 5 days of training (top panel) and the second 5 days of training (bottom panel). Solid circles indicate sequences of four sounds; open circles indicate sequences of two sounds.

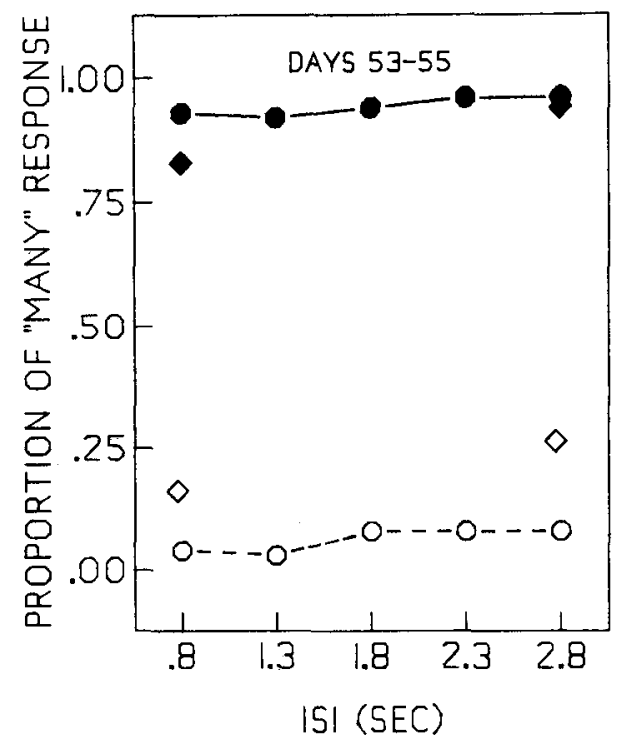

Figure 3. Median proportion of "many" responses as a function of intersound interval for the final 3 days of testing on the 14 conditions. Solid circles indicate reinforced sequences of four sounds; open circles indicate reinforced sequences of two sounds. The solid and open diamonds indicate the novel nonreinforced sequences of four sounds and two sounds, respectively.

the final 5 days of training, the greatest proportion of incorrect responses was made to the 4-sound sequence with ISI $=.8 \mathrm{sec}$, one of the three sequences used in original training.

Figure 3 shows the median probability of a "many" response during the last 3 days of testing on 14 con- ditions, in which responses to the four novel sequences (indicated by diamonds) were not reinforced. The rats continued to classify the reinforced sequences (indicated by circles) by number rather than by ISI. The slope of the regression line was not significantly different from zero for the reinforced 2 -sound sequences $[t(9)=.53, p>.05]$ or for the reinforced 4-sound sequences $[t(9)=1.82, p>.05]$.

The rats responded to the four novel sequences on the basis of the number of sounds rather than of the sound durations. The novel sequences with four sounds had a total sound-on time equal to that of the previously trained sequences with two sounds $(.4 \mathrm{sec})$, but these novel sequences were still typically classified as "many." Similarly, the novel sequences

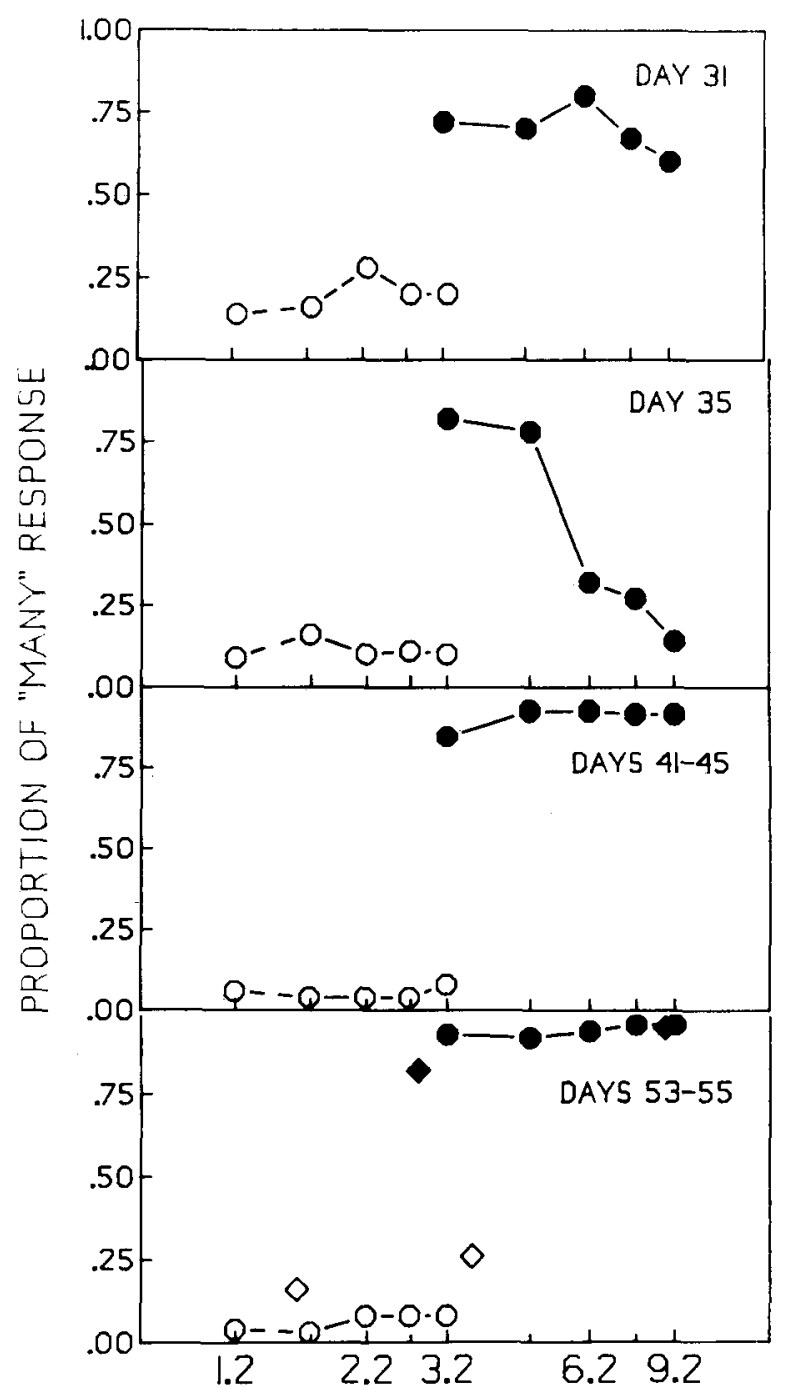

SIGNAL DURATION (SEC) ON LOGARITHMIC SCALE

Figure 4. Median proportion of "many" responses as a function of total sequence (signal) duration in logarithmic units for 1st and last days of testing, the last 5 days of training on the 10 conditions, and the final 3 days of testing on the 14 conditions. Solid circles indicate sequences of four sounds; open circles indicate sequences of two sounds. 
with two sounds had a total sound-on time equal to the previously trained sequences with four sounds $(.8 \mathrm{sec})$, but these sequences were seldom classified as "many." The percentage-correct classification of these novel 2-sound sequences was lower than that of the reinforced 2-sound sequences at the same ISI $[t(9)=4.88, p<.001]$, but the percentage-correct classification of the novel four-sound sequences was not significantly different from that of the reinforced four-sound sequences at the same ISI.

In Figure 4, the proportion of "many" responses is shown as a function of total sequence duration, that is, of time between onset of the first soundburst and termination of the final soundburst. Results for the 1st and last days of testing on the 10 conditions, the final 5 days of training on the 10 conditions, and the final 3 days of testing on the 14 conditions are shown. Time is plotted on a logarithmic scale to equalize the distances between points falling along the line for the 2-sound and 4-sound sequences; however, slope calculations are based on total duration on a linear scale. The only slope that differed significantly from zero was that for the 4-sound sequences on Day $35[t(9)=7.16$, $\mathrm{p}<.001$ ]. On the 1st day of testing (Day 31), when the total sequence durations were equal (at $3.2 \mathrm{sec}$ ), the mean proportion of "many" responses was much greater after a 4-sound sequence than after a 2-sound sequence $[\mathrm{t}(9)=6.88, \mathrm{p}<.001]$.

\section{DISCUSSION}

Using three sound sequences in initial training insured that the duration of each sound, the interval between sounds (sequence density), and the total sequence duration would not be sufficient cues for performance. The rats typically classified the sequences with two sounds in one category and the sequences with four sounds in the other. Thus, none of these temporal cues alone could determine the performance.

Of course, the animals might have learned three specific instances during initial training. However, the fact that the rats immediately classified the seven novel sequences on the basis of number indicates that they had learned something in addition to three specific instances. Although generalization may account for discrimination of the novel sequences that were similar to the trained sequences on one of the temporal dimensions, it does not count for performance on the sequence of four sounds with longer ISIs. Consider, for example, the novel 4-sound sequence with the longest ISI. The total sequence duration of this novel signal was no more like that of the trained 4-sound sequence than like that of the trained 2-sound sequences (i.e., these were both $3.2 \mathrm{sec})$, and the interval between each sound $(2.8 \mathrm{sec})$ was more like that of one of the trained 2-sound sequences (with a $2.8 \mathrm{sec}$ ISI) than like that of the trained 4-sound sequence (with a .8-sec ISI). Thus, discrimination appears to have been based on the number of successively presented sounds.

Recall, however, that throughout training the 2-short sequence was classified correctly more often than either the 2-long or the 4-long sequence. The 2-short sequence was much shorter $(1.2 \mathrm{sec})$ than either the 2-long or the 4-long sequence $(3.2 \mathrm{sec})$. Apparently, sequence duration influenced responding, although unreliably. Of course, sequence density could also have been used as an auxiliary cue in an analogous manner. The 2-long sequence was sparser (ISI $=2.8 \mathrm{sec}$ ) than either the 2-short or the 4-long sequence (ISI $=.8 \mathrm{sec}$ ). However, subjects did not appear to use this cue.

Over the 5-day test period, performance on the novel 4-sound sequences with longer ISIs declined. This is probably a consequence of nonreinforcement at all sequence durations longer than $3.2 \mathrm{sec}$ during testing, rather than of an inability to count, since later reinforcement for correct classification of these sequences restored the discrimination. During testing, the rats did not respond to these unreinforced 4-sound sequences at chance level, but consistently responded to them as "few." Perhaps the rats initially counted the sounds in these longer sequences but, as a consequence of nonreinforcement, stopped counting after a certain length of time had passed, about $3.2 \mathrm{sec}$. If subjects did, in fact, stop counting prematurely and retained this number until the levers were extended, sequences with longer ISIs would be classified more often as "few," since fewer sounds would have been presented by this time.

That the sequence most often misclassified during the last 5 days of training was one that had been used in original training (4-long) is surprising. This sequence, however, was the only 4-sound sequence that overlapped any of the 2-sound sequences in total sequence duration. Consequently, sequence duration could serve as an additional cue in classifying the other 4-sound sequences (>3.2 sec = "many").

During the first 50 sessions, the rats may have discriminated on the basis of the total sound-on time, since 2-sound sequences consisted of $.4 \mathrm{sec}$ of white noise ( 2 sounds $\times .2 \mathrm{sec}$ ) and 4-sound sequences consisted of $.8 \mathrm{sec}$ of white noise ( 4 sounds $\times .2 \mathrm{sec}$ ). The classification of the four novel sequences, in which total sound-on time was varied so that the 2-sound sequences now had total sound-on durations comparable to those of previous 4-sound sequences and vice versa, indicates that the rats were not basing their classifications on total sound-on time. Classification of these novel sequences was slightly inferior to that of the previously trained sequences, presumably because of the novelty of 
the sequences of the nonreinforcement of responses to these sequences.

The major conclusion of this study is that rats are capable of discriminating the number of sounds, even when the alternative temporal cues of duration of each sound, total sound duration, interval between each sound, and total sequence duration are controlled.

\section{REFERENCES}

Dood, P. W. Discrimination and differentiation of response number in stimulus directed pecking of pigeons. Journal of the Experimental Analysis of Behavior, 1980, 33, 253-264.

Douglas, J. W. B., \& WhITTY, C. W. M. An investigation of number appreciation in some sub-human primates. Journal of Comparative and Physiological Psychology, 1941, 31, 129-142.

Ferster, C. B., \& Skinner, B. F. Schedules of reinforcement. New York: Appleton-Century-Crofts, 1957.

HAYES, W. F. GRAPH: A BASIC-11 graphics program for digital plotters. Behavior Research Methods \& Instrumentation, 1981, $13,367$.

Hobson, S. Discriminability of fixed-ratio schedules for pigeons: Effects of absolute ratio size. Journal of the Experimental Analysis of Behavior, 1975, 23, 25-35.

Hobson, S. L., \& Newman, F. Fixed-ratio-counting schedules. In M. L. Commons \& J. A. Nevin (Eds.), Quantitative analysis of behavior (Vol. 1): Discriminative properties of reinforcement schedules. Cambridge, Mass: Ballinger, 1981.

Honigmann, H. The number concept in animal psychology. Biological Review, 1942, 17, 315-327.
LATIES, V. The modification of drug effects on behavior by external discriminative stimuli. Journal of Pharmacology and Experimental Therapeutics, 1972, 183, 1-13.

MaricQ, A. V., \& Church, R. M. The differential effects of haloperidol and methamphetamine on time estimation in the rat. Psychopharmacology, in press.

Marice, A. V., Roberts, S., \& Church, R. M. Methamphetamine and time estimation. Journal of Experimental Psychology: Animal Behavior Processes, 1981, 7, 18-30.

Mechner, F. Probability relations within response sequences maintained under ratio reinforcement. Journal of the Experimental Analysis of Behavior, 1958, 1, 109-121.

Mechner, F., \& Guevrekian, K. Effects of deprivation upon counting and timing in rats. Journal of the Experimental Analysis of Behavior, 1962, 5, 463-466.

Pliskoff, S. S., \& Goldiamond, I. Some discriminative properties of fixed-ratio performance in the pigeon. Journal of the Experimental Analysis of Behavior, 1966, 9, 1-9.

Rilling, M. Number of responses as a stimulus in fixed-ratio schedules. Journal of Comparative and Physiological Psychology, $1967,63,60-65$.

RoBerTs, S. Isolation of an internal clock. Journal of Experimental Psychology: Animal Behavior Processes, 1981, 7, 242-268.

Roberts, S., \& Chunch, R. M. Control of an internal clock. Journal of Experimental Psychology: Animal Behavior Processes, 1978, 4, 318-337.

Wilkie, D. M., Webster, J. B., \& Leader, L. G. Unconfounding time and number discrimination in a Mechner counting schedule. Bulletin of the Psychonomic Society, 1979, 13, 390-392.

(Manuscript July 29, 1981; revision accepted for publication November 30,1981 .) 